\title{
Euler's discretization, dynamic equivalence and linearization of control systems
}

\author{
Zbigniew Bartosiewicz \\ Ewa Pawłuszewicz \\ bartos@pb.bialystok.pl \\ epaw@pb.bialystok.pl \\ Institute of Mathematics \& Physics \\ Technical University of Białystok \\ Wiejska 45, 15-351 Białystok, Poland \\ tel. +48 857469073
}

\begin{abstract}
It is shown that two continuous-time control systems are dynamically feedback equivalent if and only if their Euler's discretizations are $h$-dynamically feedback equivalent for every discretization step $h$. In particular, a continuous-time system is dynamically feedback linearizable if and only if its Euler's discretization is $h$-dynamically feedback linearizable for every $h>0$. The proofs of these results are based on algebraic characterizations of dynamic feedback equivalence for continuoustime and discrete-time systems. Two continuous-time systems are dynamically feedback equivalent if and only if their differential algebras are isomorphic. Similarly, two discrete-time systems are dynamically feedback equivalent if and only if their difference algebras are isomorphic. Differential algebras corresponding to continuous-time systems and difference algebras corresponding to discretizations of those systems form two categories. Discretization induces a covariant functor from one category to the other. This functor may be inverted as the difference algebras are equipped with the whole family of difference operators corresponding to all discretizations steps $h$.
\end{abstract}

\section{Introduction}

Pawłuszewicz and Bartosiewicz (2000) have shown that if two nonlinear continuous-time systems are dynamically feedback equivalent then their Euler's discretizations are also dynamically feedback equivalent. Algebraic characterizations of the equivalence properties for both continuous-time and discrete-time systems were used. In the continuous-time case Jakubczyk $(1992,1993)$ has shown that two systems are dynamically feedback equivalent if and only if their differential algebras are isomorphic. This result has been transferred by Bar- 
tosiewicz, Jakubczyk and Pawłuszewicz (1994) to the discrete-time case, where the difference algebra of the system was used instead of differential algebra.

In both cases algebraic objects are in one-to-one correspondence with the control systems. Hence, each system may be represented by an algebraic object: differential or difference algebra. They form two categories, with morphisms preserving differential or difference structure.

In this paper we prove the converse of the result of Pawłuszewicz and Bartosiewicz (2000). Thus we show that if Euler's discretizations of two continuoustime systems are dynamically feedback equivalent for every discretization step $h$, then the continuous-time systems are dynamically feedback equivalent as well. To achieve this we have to modify the language. First, to the discretized system we associate now an algebra with a family of difference operators parameterized by $h$ and not just a single operator. Second, we slightly change the definition of this operator and the definition of dynamic feedback equivalence for discretetime systems. We have to show, however, that with this modified language we still have the same results. In fact, we generalize the result of Pawłuszewicz and Bartosiewicz (2000) to all dynamic extensions of the discrete-time control system. But the converse holds only for one of them, the one that comes from the Euler's discretization of the extension of the continuous-time system and defines $h$-dynamic feedback equivalence. Euler's discretization of a nonlinear continuous-time control system gives rise to a transformation of the differential algebra of this system into the family of difference algebras. We prove that this transformation is a covariant functor from the category $\mathcal{C}$ of the differential algebras of continuous-time systems into category $\mathcal{D}$ of the families of difference algebras parameterized by the step of discretization. We show that this functor has an inverse from $\mathcal{D}$ into $\mathcal{C}$ which gives the main result of this paper. As a byproduct we get the following statement: a continuous-time system is dynamically feedback linearizable if and only if for every discretization step $h$ its Euler's discretization is $h$-dynamically feedback linearizable.

Euler's discretization is often used to solve numerically nonlinear differential equations that describe control systems. It is important to know what happens to properties possessed by the system: which of them are invariant under the discretization and which are lost. We show here that dynamic feedback equivalence is one of the invariant properties. Another property in this context is nonsmooth (static) feedback linearization studied by Celikovský (1995).

Another method of discretization of continuous-time systems is sampling. The influence of sampling on different structural properties of systems was studied by many authors, e.g. Arapostatis et al. (1989), Grizzle and Kokotovic (1988), Kazantzis and Kravaris (1997), Kotta (1993), Monaco and NormandCyrot (1988).

The language of differential and difference algebra was first used in control theory by M. Fliess $(1986,1987,1990)$. Flies et al. (1992) also introduced the concept of flat system which is very close to a system dynamically feedback linearizable in the sense of Jakubczyk (see e.g. Pomet (1995) for the extensive discussion of these ideas and other references). 


\section{Notation}

Let $A$ be a set. By $J_{r}(A)$ we will denote the set of all sequences $Z=(z(r), z(r+$ $1), \ldots$ ) where $z(i) \in A$ and $r \in \mathbb{Z}$. We say that $Z$ starts at time $r$. If $A=$ $\mathbb{R}^{s_{1}} \times \ldots \times \mathbb{R}^{s_{k}}$, then the set $J_{r}(A)$ will be denoted by $J_{r}\left(s_{1}, \ldots, s_{k}\right)$. The union of all $J_{r}(A)$ for $r \in \mathbb{N}$ will be denoted by $J(A)$. Similarly $J\left(s_{1}, \ldots, s_{k}\right)$ will denote the union of all $J_{r}\left(s_{1}, \ldots, s_{k}\right)$ for $r \in \mathbb{N}$.

Remark 2.1. Elements of $J_{r}(s)$ will play the role of trajectories, starting at moment $r$, of discrete-time systems. Elements of $J_{0}(s)$ may be also interpreted as jets of the smooth function $t \mapsto z(t)$ at $t=0$. Thus we shall often identify $z(k)$ with the derivative $z^{(k)}(0)$. Sometimes the notation $Z=\left(z_{0}, z_{1}, \ldots\right)$ will be more suitable for $Z \in J_{0}(s)$.

The shift operator $s_{k}$ is the map $s_{k}: J_{r}(A) \rightarrow J_{r+k}(A)$ defined by $s_{k}(Z):=Y$, where $y(i)=z(i-k)$ for $i \geq r+k$ and $k \in \mathbb{Z}$. The restriction operator $c_{i}: J_{r}(A) \rightarrow J_{r+i}(A)$ is given by $c_{i}(Z):=\{z(r+i), \ldots\}, i \geq 0$.

The set $J_{0}(A)$ may be identified with the infinite product $A^{\mathbb{N}}$. If $A$ is a topological space, e.g. $\mathbb{R}^{n}$ with the standard topology, then we use the product (Tikhonov) topology on $J_{0}(A)$. The basis of this topology consists of the sets that are the inverse images of open sets in $A^{n}$ with respect to the projections $\Pi_{k}: Z \mapsto(z(0), \ldots, z(k-1))$ for $k \in \mathbb{N}$.

We shall consider real maps defined on $J(A)$. We assume that such maps are shift invariant (so we treat them as functions on $J_{0}(A)$ ) and depend on a finite number of elements $z(0), z(1), \ldots, z(q)$ of the sequence $Z \in J(A)$, but $q$ depends on the specific map. We shall call such a map finitely presented. Let $T$ be a set. A map $\phi: J(A) \rightarrow \mathbb{R}^{T}$ is finitely presented if all components of $\phi$ have this property. If $\gamma: J(A) \rightarrow B$, then the extension of $\gamma$ is the map $\Gamma: J(A) \rightarrow J(B)$, $\Gamma(Z)=\tilde{Z}, \tilde{z}(i)=\gamma\left(c_{i-r}(Z)\right)$ for $i \geq r$.

If $J_{0}(A)$ is a topological space, we consider only finitely presented continuous functions on it and the space of all these functions is equipped with the uniform convergence topology.

\section{Equivalence of systems}

We present here the definitions of dynamic feedback equivalence of nonlinear continuous-time and discrete-time systems.

\subsection{Continuous-time systems}

Let us consider an analytic or smooth continuous-time control system defined on $\mathbb{R}^{n}$ :

$$
\Sigma_{c}: \dot{x}(t)=f(x(t), u(t))
$$

where $u(t) \in \mathbb{R}^{m}$. By a trajectory of this system we will mean any pair $(x(\cdot), u(\cdot))$ that fulfils the equation (1) on some interval. The set of all trajectories of the system $\Sigma_{c}$ forms the behavior of this system. It will be denoted by $B\left(\Sigma_{c}\right)$. 
Let us consider two continuous-time systems

$$
\Sigma_{c}: \dot{x}(t)=f(x(t), u(t)) \quad \text { and } \quad \tilde{\Sigma}_{c}: \dot{\tilde{x}}(t)=\tilde{f}(\tilde{x}(t), \tilde{u}(t)
$$

where $x(t) \in \mathbb{R}^{n}, \tilde{x}(t) \in \mathbb{R}^{\tilde{n}}, u(t), \tilde{u}(t) \in \mathbb{R}^{m}, t \in \mathbb{R}$. Following Jakubczyk (1992) we say that $\Sigma_{c}$ and $\tilde{\Sigma}_{c}$ are dynamically feedback equivalent if there exist transformations:

$$
\begin{array}{ll}
x=\phi(\tilde{X}), & u=\psi(\tilde{X}, \tilde{U}) \\
\tilde{x}=\tilde{\phi}(X), & \tilde{u}=\tilde{\psi}(X, U)
\end{array}
$$

where $\phi: J(\tilde{n}) \rightarrow \mathbb{R}^{n}, \tilde{\phi}: J(n) \rightarrow \mathbb{R}^{\tilde{n}}, \psi: J(\tilde{n}, \tilde{m}) \rightarrow \mathbb{R}^{m}, \tilde{\psi}: J(n, m) \rightarrow \mathbb{R}^{\tilde{n}}$ are finitely presented maps of class $C^{s}, s=\omega$ or $s=\infty$, such that the induced maps on pairs $(x(\cdot), u(\cdot))$ and $(\tilde{x}(\cdot), \tilde{u}(\cdot))$ transform behaviors of one system onto behaviors of the second one and are mutually inverse on these behaviors. Thus we get

$$
\begin{aligned}
& x(t)=\phi\left(\tilde{x}(t), \tilde{x}^{\prime}(t), \ldots, \tilde{x}^{(k)}(t)\right) \\
& u(t)=\psi\left(\tilde{x}(t), \tilde{x}^{\prime}(t), \ldots, \tilde{x}^{(k)}(t), \tilde{u}(t), \tilde{u}^{\prime}(t), \ldots, \tilde{u}^{(k)}(t)\right)
\end{aligned}
$$

for some $k \geq 0$, and similarly for $(\tilde{x}(t), \tilde{u}(t))$. System $\Sigma_{c}$ is dynamically feedback linearizable if it is dynamically feedback equivalent to a linear controllable one.

\subsection{Discrete-time systems}

Let us consider a nonlinear discrete-time control system of class $C^{s}$ defined on $\mathbb{R}^{n}$ :

$$
\Sigma_{d}: x(k+1)=g(x(k), u(k))
$$

where $u(k) \in \mathbb{R}^{m}, k \in \mathbb{Z}$ and $g$ is of class $C^{s}$.

Let $H: J_{0}(m) \rightarrow J_{0}(m)$ be a finitely presented map of class $C^{s}$. If necessary, we can extend it to sequences starting at arbitrary time $r$ putting $H(U)=$ $s_{r}\left(H\left(s_{-r}(U)\right)\right)$.

By the $H$-extension of $\Sigma_{d}$ we mean the system $\Sigma_{d}^{H}$ defined by

$$
\begin{aligned}
x(k+1) & =g\left(x(k), u_{0}(k)\right) \\
U(k+1) & =H(U(k)),
\end{aligned}
$$

where $U(k)=\left(u_{0}(k), u_{1}(k), \ldots\right) \in J_{0}(m)$.

Let us consider the sequences starting at time $r: X=\{x(r), x(r+1), \ldots\} \in$ $J_{r}(n)$ and $\left.U=\{U(r), U(r+1), \ldots\} \in J_{r}\left(J_{0}(m)\right)\right)$. The couple $Z=(X, U)$ is a $H$-trajectory (starting at time $r$ ) of the system $\Sigma_{d}$ if it satisfies (5) for $k \geq r$. We shall often identify $Z$ with the sequence $(Z(r), Z(r+1), \ldots)$, where $Z(k)=(x(k), U(k))$. The set of all $H$-trajectories of the system $\Sigma_{d}$ forms the $H$-behavior of this system. We will denote it by $B^{H}\left(\Sigma_{d}\right)$. Moreover, $B^{H}\left(\Sigma_{d}\right)=$ $\bigcup_{r \in Z} B_{r}^{H}\left(\Sigma_{d}\right)$, where $B_{r}^{H}\left(\Sigma_{d}\right)$ is the set of all $H$-trajectories starting at time $r$. 
Let $Z=(X, U)$ be a $H$-trajectory starting at time 0 with initial condition $(x(0), U(0))$. We define $P=(T, R)$ as the map $P(x(0), U(0))=(X, U)$. We shall assume that

A0. $T$ is left invertible, i.e. we can recover $(x(0), U(0))$ from $X$. Then $P$ is also left invertible.

Let us consider two nonlinear discrete-time systems defined, respectively, on $\mathbb{R}^{n}$ and $\mathbb{R}^{\tilde{n}}$ :

$$
\Sigma_{d}: x(k+1)=g(x(k), u(k)) \text { and } \tilde{\Sigma}_{d}: \tilde{x}(k+1)=\tilde{g}(\tilde{x}(k), \tilde{u}(k))
$$

where $u(k), \tilde{u}(k) \in \mathbb{R}^{m}$. Let us consider finitely presented maps:

$$
\phi: J(\tilde{n}) \rightarrow \mathbb{R}^{n}, \Psi: J(\tilde{n}, m) \rightarrow J_{0}(m) \text { and } \tilde{\phi}: J(n) \rightarrow \mathbb{R}^{\tilde{n}}, \tilde{\Psi}: J(n, m) \rightarrow J_{0}(m) .
$$

They define dynamic transformations of states and extended controls of both systems of the form:

$$
\begin{aligned}
x(k) & =\phi(\tilde{x}(k), \ldots, \tilde{x}(k+\tilde{q})) \\
u_{i}(k) & =\Psi_{i}\left(\tilde{x}(k), \ldots, \tilde{x}\left(k+\tilde{q}_{i}\right), \tilde{u}_{0}(k), \ldots, \tilde{u}_{\tilde{q}_{i}}(k)\right)
\end{aligned}
$$

and

$$
\begin{aligned}
\tilde{x}(k) & =\tilde{\phi}(x(k), \ldots, x(k+q)) \\
\tilde{u}_{i}(k) & =\tilde{\Psi}\left(x(k), \ldots, x\left(k+q_{i}\right), u_{0}(k), \ldots, u_{q_{i}}(k)\right)
\end{aligned}
$$

Let $\Phi$ and $\tilde{\Phi}$ be the extensions of $\phi$ and $\tilde{\phi}$, respectively. By $H$-extension of $\Psi$ we mean the map $\Psi^{H}: J(\tilde{n}) \times J\left(J_{0}(m)\right) \rightarrow J\left(J_{0}(m)\right)$ defined by

$$
\Psi_{k}^{H}(\tilde{X}, \tilde{U})=\Psi\left(c_{k}(\tilde{X}), H^{k}(\tilde{U})\right),
$$

for $\tilde{X}$ and $\tilde{U}$ starting at 0 and $k \geq 0$. Similarly $\tilde{\Psi}^{H}$ is defined.

Let $\chi: J(\tilde{n}) \times J\left(J_{0}(m)\right) \rightarrow J(n) \times J\left(J_{0}(m)\right)$ and $\tilde{\chi}: J(n) \times J\left(J_{0}(m)\right) \rightarrow J(\tilde{n}) \times$ $J\left(J_{0}(m)\right)$ be defined as follows:

$$
\chi=\left(\Phi, \Psi^{H}\right), \quad \tilde{\chi}=\left(\tilde{\Phi}, \tilde{\Psi}^{H}\right) .
$$

We say that systems $\Sigma_{d}$ and $\tilde{\Sigma}_{d}$ are $H$-dynamically feedback equivalent if there exist maps $(\phi, \Psi)$ and $(\tilde{\phi}, \tilde{\Psi})$ such that

$$
\chi\left(B^{H}\left(\tilde{\Sigma}_{d}\right)\right)=B^{H}\left(\Sigma_{d}\right), \quad \tilde{\chi}\left(B^{H}\left(\Sigma_{d}\right)\right)=B^{H}\left(\tilde{\Sigma}_{d}\right)
$$

and $\chi$ and $\tilde{\chi}$ are mutually inverse on the systems' $H$-behaviors.

Discrete-time system $\Sigma_{d}$ is $H$-dynamically feedback linearizable if it is $H$ dynamically feedback equivalent to a controllable linear one.

Remark 3.1. The definition of $H$-dynamical feedback equivalence requires maps $\Psi$ and $\tilde{\Psi}$ with values in infinite-dimensional spaces. This is the cost we have to pay for a more general definition than in the continuous-time case. However in 
many cases $\Psi$ may be regarded as an infinite extension of a finite-dimensional map.

Two particular cases of $H$ will be important in our studies: $H(U)=c_{1}(U)$ and $H_{h}(U)=U+h c_{1}(U)$, where $U \in J_{0}(m)$ and $h>0$. In the first case $\Psi$ may be redefined as the extension of $\psi=\Psi_{0}$ :

$$
\Psi(\tilde{X}, \tilde{U})=\left(\psi(\tilde{X}, \tilde{U}), \psi\left(c_{1}(\tilde{X}), c_{1}(\tilde{U})\right), \ldots\right)
$$

In the second case, the components of $\Psi$ may be defined recurrently: $\Psi_{k+1}(\tilde{X}, \tilde{U})=$ $\left(\Psi_{k}\left(c_{1}(\tilde{X}), H(\tilde{U})\right)-\Psi_{k}(\tilde{X}, \tilde{U})\right) / h$, so again it is enough to have the finitedimensional map $\psi=\Psi_{0}$. Observe that for both cases the map $T$ which assigns $X$ to the initial condition $(x(0), U(0))$ is left invertible.

We say that two discrete-time systems are $h$-dynamically feedback equivalent if they are $H_{h}$-dynamically feedback equivalent. Similarly we define $h$-dynamic feedback linearizability of a discrete-time system.

Remark 3.2. Bartosiewicz, Jakubczyk and Pawłuszewicz (1994) introduced the anticipating feedback equivalence. It is exactly $H$-dynamic feedback equivalence for $H(U)=c_{1}(U), U \in J_{0}(m)$. In general, two $H$-dynamically feedback equivalent systems do not have to be $\tilde{H}$-dynamically feedback equivalent for another map $\tilde{H}$. The concept of $H$-dynamic feedback equivalence could be also introduced for continuous-time systems. But it will only be used in the discrete-time case to deal with the discretized systems, so there is no need of a more general approach.

\section{Differential and difference algebras}

A differential algebra is a commutative algebra $\mathcal{A}$ over $\mathbb{R}$ together with a differential operator $D: \mathcal{A} \rightarrow \mathcal{A}$, i.e. a linear map satisfying the Leibniz rule for product. A map $\tau: \mathcal{A}_{1} \rightarrow \mathcal{A}_{2}$ is a homomorphism of differential algebras $\left(\mathcal{A}_{1}, D_{1}\right)$ and $\left(\mathcal{A}_{2}, D_{2}\right)$ if it is a homomorphism of algebras and $D_{2} \circ \tau=\tau \circ D_{1}$.

A difference algebra is a commutative algebra $\mathcal{A}$ over $\mathbb{R}$ together with a homomorphism $d: \mathcal{A} \rightarrow \mathcal{A}$, called difference operator. If $\left(\mathcal{A}_{1}, d_{1}\right),\left(\mathcal{A}_{2}, d_{2}\right)$ are two difference algebras then a map $\tau: \mathcal{A}_{1} \rightarrow \mathcal{A}_{2}$ is a homomorphism of these algebras if it is homomorphism of algebras and $d_{2} \circ \tau=\tau \circ d_{1}$. If the homomorphism $\tau$ is a bijective map, then $\tau$ is an isomorphism of, respectively, differential or difference algebras.

A parametric difference algebra is a commutative algebra $A$ over $\mathbb{R}$ with a family $d=\left\{d_{s}, s \in S\right\}$ of difference operators, where $S$ is the set of parameters. Let $\left(\mathcal{A}_{1}, d^{1}\right),\left(\mathcal{A}_{2}, d^{2}\right)$ be two parametric difference algebras with the same set of parameters. A map $\tau: \mathcal{A}_{1} \rightarrow \mathcal{A}_{2}$ is a homomorphism (isomorphism) of these algebras if for every $s \in S$ it is a homomorphism (isomorphism) of difference algebras $\left(A_{1}, d_{s}^{1}\right) \rightarrow\left(A_{2}, d_{s}^{2}\right)$. 
Let $\mathcal{A}(n, m)$ denote the algebra of all finitely presented, shift invariant, real functions of class $C^{s}$ defined on $\mathbb{R}^{n} \times J(m)$. We may treat them as functions on $\mathbb{R}^{n} \times J_{0}(m)$. We will assume that functions in $\mathcal{A}(n, m)$ are of the same class as the dynamics $f$ of the continuous- or discrete-time system that is considered.

From now on, in both continuous-time and discrete-time cases we will assume the following:

A1. For every $x, y \in \mathbb{R}^{n}$ there is at most one $u$ that satisfies the equation $y=f(x, u)$.

A2. For any $x$ and $u$ the rank of matrix $\frac{\partial f}{\partial u}(x, u)$ is full.

A3. The map $\mathbb{R}^{n} \times \mathbb{R}^{m} \rightarrow \mathbb{R}^{n} \times \mathbb{R}^{m}:(x, u) \mapsto(x, f(x, u))$ is proper.

By the differential operator associated with the system $\Sigma_{c}$ we will mean the map $D_{\Sigma_{c}}: \mathcal{A}(n, m) \rightarrow \mathcal{A}(n, m)$ introduced by Jakubczyk (1992):

$$
D_{\Sigma_{c}}:=\sum_{1 \leq q \leq n} f_{q} \frac{\partial}{\partial x_{q}}+\sum_{i, j} u_{j}^{(i+1)} \frac{\partial}{\partial u_{j}^{(i)}}
$$

where $U=\left(\left(u_{j}^{(i)} ; j=1, \ldots, m\right) ; i \geq 0\right) \in J_{0}(m)$. The second sum is treated as a formal sum. For $\varphi \in \mathcal{A}(n, m), D_{\Sigma_{c}} \varphi$ is a well defined, finitely presented function. If $l \geq 2$, then $D_{\Sigma_{c}}^{l} \varphi:=D_{\Sigma_{c}}\left(D_{\Sigma_{c}}^{l-1} \varphi\right)$. The algebra $\mathcal{A}(n, m)$ together with the differential operator $D_{\Sigma_{c}}$ forms a differential algebra called the differential algebra of the system $\Sigma_{c}$ and denoted by $\left(\mathcal{A}(n, m), D_{\Sigma_{c}}\right)$ or shortly by $\mathcal{A}_{\Sigma_{c}}$. Jakubczyk (1992) proved the following result.

Theorem 4.1. Under assumptions A1 - A3, two analytic (or smooth) systems $\Sigma_{c}$ and $\tilde{\Sigma}_{c}$ are dynamically feedback equivalent if and only if their differential algebras are isomorphic.

The differential operator $D_{\Sigma_{c}}$ may be interpreted as an abstract vector field on the infinite-dimensional space $\mathbb{R}^{n} \times J_{0}(m)$. The vector field, in turn, corresponds to the following infinite system of differential equations

$$
\begin{aligned}
\dot{x} & =f\left(x, u_{0}\right) \\
\dot{u}_{0} & =u_{1} \\
\dot{u}_{1} & =u_{2} \\
\ldots & \ldots
\end{aligned}
$$

The difference operator corresponding to the discrete-time system $\Sigma_{d}$ will depend on the map $H$ used to extend the system. The difference operator associated with $\Sigma_{d}$ is given by the formula

$$
\left(d_{\Sigma_{d}}^{H} \varphi\right)(x, U)=\varphi\left(g\left(x, u_{0}\right), H(U)\right),
$$

where $U=\left(u_{0}, u_{1}, \ldots\right)$. The difference algebra $\mathcal{A}_{\Sigma_{d}}^{H}=\left(\mathcal{A}(n, m), d_{\Sigma_{d}}^{H}\right)$ will be called $H$-difference algebra of $\Sigma_{d}$. When we evaluate $\varphi$ on a $H$-trajectory of $\Sigma_{d}$ at time $k,(x(k), U(k))$, then its time shift is described by $d_{\Sigma_{d}}^{H}$ :

$$
\varphi(x(k+1), U(k+1))=\left(d_{\Sigma_{d}}^{H} \varphi\right)(x(k), U(k)) .
$$


The most natural extension is given by $H=c_{1}$. It leads to the following

$$
\begin{aligned}
x(k+1) & =g\left(x(k), u_{0}(k)\right) \\
u_{0}(k+1) & =u_{1}(k) \\
u_{1}(k+1) & =u_{2}(k)
\end{aligned}
$$

where the components of $U \in J_{0}(m)$ are interpreted as subsequent time shifts of the control $u=u_{0}$.

This approach was used by Bartosiewicz, Jakubczyk and Pawłuszewicz (1994) to present a characterization of the dynamic (or anticipating) feedback equivalence in the discrete-time case. We need an extension of this fact to arbitrary $H$-dynamic feedback equivalence.

Theorem 4.2. Under assumptions A0-A3, two discrete time systems are $H$ dynamically feedback equivalent if and only if their $H$-difference algebras are isomorphic.

Proof. " $\Rightarrow$ " Assume that $\Sigma$ and $\tilde{\Sigma}$ are $H$-dynamically feedback equivalent. Then the maps $\chi$ and $\tilde{\chi}$ corresponding to dynamic transformations transform a $H$ trajectory of one system onto a $H$-trajectory of the other system. Let $Z(k)=$ $(x(k), U(k))$ and $\tilde{Z}(k)=(\tilde{x}(k), \tilde{U}(k))$ denote $H$-trajectories evaluated at time $k$. Let $g^{H}(x, U)=\left(g\left(x, u_{0}\right), H(U)\right)$ and $\tilde{g}^{H}(\tilde{x}, \tilde{U})=\left(\tilde{g}\left(\tilde{x}, \tilde{u}_{0}\right), H(\tilde{U})\right)$. Then $d_{\Sigma_{d}}^{H}(\varphi)=\varphi \circ g^{H}$. For every $Z(0)$ we get

$$
\begin{gathered}
\left(\tilde{P}^{-1} \circ \tilde{\chi} \circ P \circ g^{H}\right)(Z(0))=\tilde{P}^{-1}(\tilde{\chi}(P(Z(1))))=\tilde{Z}(1)=\tilde{g}^{H}(\tilde{Z}(0)) \\
=\tilde{g}^{H}\left(\tilde{P}^{-1}(\tilde{\chi}(P(Z(0))))\right)=\left(\tilde{g}^{H} \circ \tilde{P}^{-1} \circ \tilde{\chi} \circ P\right)(Z(0)) .
\end{gathered}
$$

Let $\tilde{\tau}=\left(\tilde{P}^{-1} \circ \tilde{\chi} \circ P\right)^{*}$, i.e. $\tilde{\tau}(\tilde{\varphi})=\tilde{\varphi} \circ \tilde{P}^{-1} \circ \tilde{\chi} \circ P$ for $\tilde{\varphi} \in \mathcal{A}_{\tilde{\Sigma}_{d}}^{H}$. Observe that $\tilde{P}^{-1}$ acts on $H$-trajectories, so the expression is well defined. Hence, from (10)

$$
\begin{aligned}
d_{\Sigma_{d}}^{H}(\tilde{\tau} \tilde{\varphi})= & \tilde{\varphi} \circ \tilde{P}^{-1} \circ \tilde{\chi} \circ P \circ g^{H}=\tilde{\varphi} \circ \tilde{g}^{H} \circ \tilde{P}^{-1} \circ \tilde{\chi} \circ P \\
& =\left(d_{\tilde{\Sigma}_{d}}^{H} \tilde{\varphi}\right) \circ \tilde{P}^{-1} \circ \tilde{\chi} \circ P=\tilde{\tau}\left(d_{\tilde{\Sigma}_{d}}^{H} \tilde{\varphi}\right),
\end{aligned}
$$

which means that $\tilde{\tau}$ is a homomorphism of difference algebras $\mathcal{A}_{\tilde{\Sigma}_{d}}^{H}$ and $\mathcal{A}_{\Sigma_{d}}^{H}$. Similarly we show that $\tau=\left(P^{-1} \circ \chi \circ \tilde{P}\right)^{*}$ is a homomorphism from $\mathcal{A}_{\Sigma_{d}}^{H}$ to $\mathcal{A}_{\tilde{\Sigma}_{d}}^{H}$. As the compositions $\chi \circ \tilde{\chi}$ and $\tilde{\chi} \circ \chi$ are identities on the behaviors we get that $\tau \circ \tilde{\tau}=\left(P^{-1} \circ \chi \circ \tilde{P}\right)^{*} \circ\left(\tilde{P}^{-1} \circ \tilde{\chi} \circ P\right)^{*}=\left(\tilde{P}^{-1} \circ \tilde{\chi} \circ P \circ P^{-1} \circ \chi \circ \tilde{P}\right)^{*}=$ id and similarly for $\tilde{\tau} \circ \tau$. This means that $\tau$ and $\tilde{\tau}$ are isomorphisms of the difference algebras.

"६" Assume that $\tau: \mathcal{A}_{\Sigma_{d}}^{H} \rightarrow \mathcal{A}_{\tilde{\Sigma}_{d}}^{H}$ is an isomorphism of $H$-difference algebras. Let $x=\left(x^{1}, \ldots, x^{n}\right)$ and $U=\left(u_{01}, \ldots, u_{0 m}, \ldots\right)$ be treated as sequences of functions from $A(n, m)$. Then we define $\phi(\tilde{X})=(\tau(x))\left(\tilde{T}^{-1}(\tilde{X})\right)$ and $\Psi(\tilde{X}, \tilde{U})=$ $(\tau(U))\left(\tilde{T}^{-1}(\tilde{X})\right)$, where $\tilde{X}$ is the first component of an $H$-trajectory $Z$ and $\tilde{U} \in$ $J_{0}(m)$ is arbitrary (actually $\tilde{U}$ is redundant here because of A0). Thus we have 
$\tau(x)=\phi \circ \tilde{T}=x \circ \phi \circ \tilde{T}=(\phi \circ \tilde{T})^{*}(x)$ and $\tau(U)=\Psi \circ \tilde{T}=U \circ \Psi \circ \tilde{T}=(\Psi \circ \tilde{T})^{*}(U)$. By the result of Jakubczyk (1992), this means that $\tau=(\phi \circ \tilde{T}, \Psi \circ \tilde{T})^{*}$ on the entire algebra $\mathcal{A}(\tilde{n}, m)$.

Let $\tilde{X}=(\tilde{x}(0), \tilde{x}(1), \ldots)$ and $\tilde{U}=(\tilde{U}(0), \tilde{U}(1), \ldots)$ form a $H$-trajectory of $\tilde{\Sigma}_{d}$ starting at 0 . Let $x(k)=\phi\left(c_{k}(\tilde{X})\right)$ and $U(k)=\Psi\left(c_{k}(\tilde{X}), \tilde{U}(k)\right)$. We are going to show that $X=(x(0), x(1), \ldots)$ and $U=(U(0), U(1), \ldots)$ form a $H$-trajectory of $\Sigma_{d}$ :

$$
\begin{gathered}
x(k+1)=\phi\left(c_{k+1}(\tilde{X})\right)=(\tau(x))(\tilde{x}(k+1), \tilde{U}(k+1)) \\
=(\tau(x))\left(\tilde{g}^{H}(\tilde{x}(k), \tilde{U}(k))=\left(d_{\tilde{\Sigma}_{d}}^{H}(\tau(x))(\tilde{x}(k), \tilde{U}(k))=\tau\left(d_{\Sigma_{d}}^{H}(x)\right)(\tilde{x}(k), \tilde{U}(k))\right.\right. \\
=\tau(g)(\tilde{x}(k), \tilde{U}(k))=(g \circ(\phi \circ \tilde{T}, \Psi \circ \tilde{T}))(\tilde{x}(k), \tilde{U}(k)) \\
=g(x(k), U(k))=g\left(x(k), u_{0}(k)\right) .
\end{gathered}
$$

Similarly

$$
\begin{gathered}
U(k+1)=\Psi\left(c_{k+1} \tilde{X}, \tilde{U}(k+1)\right)=(\tau(U))(\tilde{x}(k+1), \tilde{U}(k+1)) \\
=(\tau(U))\left(\tilde{g}^{H}(\tilde{x}(k), \tilde{U}(k))=\left(d_{\tilde{\Sigma}_{d}}^{H}(\tau(U))(\tilde{x}(k), \tilde{U}(k))=\tau\left(d_{\Sigma_{d}}^{H}(U)\right)(\tilde{x}(k), \tilde{U}(k))\right.\right. \\
=\tau(H)(\tilde{x}(k), \tilde{U}(k))=(H \circ(\phi \circ \tilde{T}, \Psi \circ \tilde{T}))(\tilde{x}(k), \tilde{U}(k))=H(U(k)) .
\end{gathered}
$$

Thus $\phi$ and $\Psi$ define a transformation that transports $H$-trajectories of one system onto $H$-trajectories of the other. To extend $\phi$ and $\Psi$ to $J_{0}(\tilde{n})$ and $J_{0}(\tilde{n}, m)$, respectively, we proceed as Bartosiewicz, Jakubczyk and Pawłuszewicz (1994), using assumption A3.

Let $h>0$. By the Euler's discretization associated with the system $\Sigma_{c}$, corresponding to $h$, we will mean the system $\Sigma_{d}^{h}$ defined by

$$
x(k+1)=x(k)+h f(x(k), u(k))
$$

If we extend (12) using the operator $H$, then the difference operator $d_{h}^{H}$ associated to (12) will take on the form

$$
\left(d_{h}^{H} \varphi\right)(x, U)=\varphi\left(x+h f\left(x, u_{0}\right), H(U)\right) .
$$

The map $H=c_{1}$ was used by Bartosiewicz and Pawłuszewicz (2000) to prove that if two continuous-time systems are dynamically feedback equivalent, then their Euler's discretizations are $c_{1}$-dynamically feedback equivalent. The following theorem gives a generalization of that result.

Theorem 4.3. If two continuous-time systems are dynamically feedback equivalent, then for every discretization step $h$ and for every map $H$ the Euler's discretizations of these systems corresponding to $h$ are $H$-dynamically feedback equivalent.

The proof of Theorem 4.3 is a consequence of Theorems 4.1 and 4.2 and the following lemma. 
Lemma 4.4. Let $\tau$ be a homomorphism of the differential algebras

$$
\tau:\left(\mathcal{A}(n, m), D_{\Sigma_{c}}\right) \rightarrow\left(\mathcal{A}(\tilde{n}, m), D_{\tilde{\Sigma}_{c}}\right) .
$$

Then for every $h$ and $H, \tau$ is a homomorphism of the difference algebras $\tau$ : $\left(\mathcal{A}(n, m), d_{h}^{H}\right) \rightarrow\left(\mathcal{A}(\tilde{n}, m), \tilde{d}_{h}^{H}\right)$ of the discretized systems.

Proof. From the assumption we have $\tau \circ D_{\Sigma_{c}}=D_{\tilde{\Sigma}_{c}} \circ \tau$. We shall show that $\tau \circ d_{h}^{H}=\tilde{d}_{h}^{H} \circ \tau$.

Let $\tilde{\pi}_{1}$ and $\tilde{\pi}_{2}$ be the projections $(\tilde{x}, \tilde{U}) \mapsto \tilde{x}$ and $(\tilde{x}, \tilde{U}) \mapsto \tilde{U}$, respectively. It was shown by Jakubczyk (1992) that for the map $\tau$ there exists a map $\mu$ : $\mathbb{R}^{\tilde{n}} \times J_{0}(m) \rightarrow \mathbb{R}^{n} \times J_{0}(m)$ such that $\tau=\mu^{*}$. Let $F: R^{k} \rightarrow \mathbb{R}$, then

$$
\tau\left(F\left(\varphi_{1}, \ldots, \varphi_{k}\right)=F\left(\varphi_{1} \circ \mu, \ldots, \varphi_{k} \circ \mu\right)=F\left(\tau \varphi_{1}, \ldots, \tau \varphi_{k}\right)\right.
$$

Hence $\tau$ commutes with substitutions. In particular, $\tau \varphi=\tau(\varphi \circ(x, U))=$ $\varphi(\tau x, \tau U)$ and $\tau(H)=H(\tau U)$ which gives

$$
\begin{aligned}
\tilde{d}_{h}^{H}(\tau \varphi) & =(\tau \varphi)\left(\left(\mathrm{id}+h D_{\tilde{\Sigma}_{c}}\right) \circ \tilde{\pi}_{1}, H \circ \tilde{\pi}_{2}\right) \\
& =\varphi\left(\tau\left(\left(\mathrm{id}+h D_{\tilde{\Sigma}_{c}}\right) \circ \tilde{\pi}_{1}, H \circ \tilde{\pi}_{2}\right)\right) \\
& =\varphi\left(\left(\mathrm{id}+h D_{\Sigma_{c}}, H\right)\left(\tau\left(\tilde{\pi}_{1}, \tilde{\pi}_{2}\right)\right)\right) \\
& =\left(d_{h}^{H} \varphi\right)\left(\tau\left(\tilde{\pi}_{1}, \tilde{\pi}_{2}\right)=\tau\left(d_{h}^{H} \varphi\right) .\right.
\end{aligned}
$$

The converse of Theorem 4.3 will be proved in the next section only for a particular choice of the map $H$, involving the discretization step $h$. This $H$ is related to the discretization of the extended system (7) which gives the following extension of (12).

$$
\begin{aligned}
x(k+1) & =x(k)+h f\left(x(k), u_{0}(k)\right) \\
u_{0}(k+1) & =u_{0}(k)+h u_{1}(k) \\
u_{1}(k+1) & =u_{1}(k)+h u_{2}(k)
\end{aligned}
$$

Thus in this case $H_{h}(U)=U+h c_{1}(U)$ naturally appears. Now the difference operator, denoted by $d_{h}$, corresponding to $\Sigma_{d}^{h}$ is as follows:

$$
\left(d_{h} \varphi\right)(x, U)=\varphi\left(x+h f\left(x, u_{0}\right), U+h c_{1}(U)\right) .
$$

where $\varphi \in \mathcal{A}(n, m)$ and $U=\left(u_{0}, u_{1}, \ldots\right)$ as before. If $l \geq 2$, then $d_{h}^{l} \varphi:=$ $d_{h}\left(d_{h}^{l-1}\right) \varphi$.

The algebra $\mathcal{A}(n, m)$ together with the family of difference operators $d=$ $\left\{d_{h}, h>0\right\}$, is a parametric difference algebra (parameterized by $h>0$ ). We will call it the parametric difference algebra of the system $\Sigma_{d}$ (with the family of difference operators $d_{h}$ ), and denote by $\mathcal{A}_{\Sigma_{d}}$. 


\section{Categories of differential and difference alge- bras}

We define the category $\mathcal{C}$ of differential algebras of continuous-time systems as follows:

1. by the class of objects $\mathrm{ObC}$ of $\mathcal{C}$ we will mean the class of all differential algebras $\mathcal{A}_{\Sigma_{c}}$ corresponding to continuous-time systems $\Sigma_{c}$.

2 . by the set of morphisms of $\mathcal{C}$, denoted by $\operatorname{Mor} \mathcal{C}$, we will mean the set of all homomorphisms between differential algebras from $\mathrm{ObC}$.

Let $\left(\mathcal{A}_{1}, D_{1}\right),\left(\mathcal{A}_{2}, D_{2}\right),\left(\mathcal{A}_{3}, D_{3}\right) \in \mathrm{ObC}$ and $\nu:\left(\mathcal{A}_{1}, D_{1}\right) \rightarrow\left(\mathcal{A}_{2}, D_{2}\right), \tau:$ $\left(\mathcal{A}_{2}, D_{2}\right) \rightarrow\left(\mathcal{A}_{3}, D_{3}\right)$ be morphisms from MorC. Then $\nu \circ \tau \in \operatorname{Mor} \mathcal{C}, \nu \circ \tau$ : $\left(\mathcal{A}_{1}, D_{1}\right) \rightarrow\left(\mathcal{A}_{3}, D_{3}\right)$, is the composition of morphisms. The identity map $\mathcal{A} \rightarrow \mathcal{A}$ is the unit morphism.

Now we are going to define the category $\mathcal{D}$ of parametric difference algebras of discrete-time systems that are the Euler's discretizations of continuous-time systems. We define the category $\mathcal{D}$ as follows:

1. the class of objects $\mathrm{ObD}$ of category $\mathcal{D}$ is formed by the parametric difference algebras $\mathcal{A}_{\Sigma_{d}}$ (parameterized by $h$ ) corresponding to the discretizations (14) of the continuous-time system $\Sigma_{c}$.

2. the class of morphisms MorD of $\mathcal{D}$ consists of all homomorphisms between parametric difference algebras from $\mathrm{ObD}$. Composition of morphisms and the unit morphism are defined as before.

Let $\mathcal{F}: \mathcal{C} \rightarrow \mathcal{D}$ be a functor from category $\mathcal{C}$ into category $\mathcal{D}: \mathcal{F}:=\left(\mathcal{F}_{1}, \mathcal{F}_{2}\right)$, $\mathcal{F}_{1}: \operatorname{ObC} \rightarrow \mathrm{ObD}, \mathcal{F}_{2}: \operatorname{Mor} \mathcal{C} \rightarrow \operatorname{Mor} \mathcal{D}$. Let $\mathcal{F}_{1}(\mathcal{A}(n, m)):=\mathcal{A}(n, m)$ and let $\mathcal{F}_{1} D_{\Sigma_{c}}$ be a family of difference operators, parameterized by $h$, defined by

$$
\begin{array}{r}
\left(\mathcal{F}_{1} D_{\Sigma_{c}}\right)_{h} x:=\left(\mathrm{id}+h D_{\Sigma_{c}}\right) x=x+h f\left(x, u_{0}\right) \\
\left(\mathcal{F}_{1} D_{\Sigma_{c}}\right)_{h} U:=\left(\mathrm{id}+h D_{\Sigma_{c}}\right) U=U+h c_{1}(U)
\end{array}
$$

where $x=\left(x_{1}, \ldots, x_{n}\right)$ and $U=\left(u_{0}, u_{1}, \ldots\right)$ and

$$
\left(\mathcal{F}_{1} D_{\Sigma_{c}}\right)_{h} \varphi(x, U):=\varphi\left(\left(\mathcal{F}_{1} D_{\Sigma_{c}}\right)_{h} x,\left(\mathcal{F}_{1} D_{\Sigma_{c}}\right)_{h} U\right)
$$

for any $\varphi \in \mathcal{A}(n, m)$ and $h>0$. We treat $x$ and $U$ as maps $x: \mathbb{R} \times J(m) \rightarrow \mathbb{R}^{n}$ and $U: \mathbb{R} \times J(m) \rightarrow J(m)$, respectively, so $x$ and $U$ are collections of finitely presented functions from $\mathcal{A}(n, m)$. Above id denotes the identity map on $\mathcal{A}(n, m)$. Since $D_{\Sigma_{c}} x=f$, the difference operator $d_{h}=\left(\mathcal{F}_{1} D_{\Sigma_{c}}\right)_{h}$ may be written as $d_{h} \varphi(x, U)=\varphi\left(x+h f(x, u), U+h c_{1}(U)\right)$ where $u=u(0)$. It corresponds to the discrete-time system (14). It is the Euler's discretization of (7) with the step $h$ and $H(U)=U+h c_{1}(U)$.

Finally, if $\tau: \mathcal{A}_{1} \rightarrow \mathcal{A}_{2}$ is a homomorphism of differential algebras $\left(\mathcal{A}_{1}, D_{1}\right)$ and $\left(\mathcal{A}_{2}, D_{2}\right)$ from $\mathrm{ObC}$, then $\mathcal{F}_{2}(\tau):=\tau$ as a homomorphism of algebras (recall that $\mathcal{F}_{1}\left(\mathcal{A}_{1}\right)=\mathcal{A}_{1}$ and $\left.\mathcal{F}_{1}\left(\mathcal{A}_{2}\right)=\mathcal{A}_{2}\right)$. From Lemma 4.4 we obtain that $\tau$ is then a homomorphism of the difference algebras $\left(A_{1}, \mathcal{F}_{1}\left(D_{1}\right)\right)$ and $\left(A_{2}, \mathcal{F}_{1}\left(D_{2}\right)\right)$. Thus we get 
Theorem 5.1. $\mathcal{F}$ is a covariant functor from category $\mathcal{C}$ to category $\mathcal{D}$.

Let us consider now a functor $\mathcal{G}=\left(\mathcal{G}_{1}, \mathcal{G}_{2}\right)$ from category $\mathcal{D}$ to category $\mathcal{C}$, defined by: $\mathcal{G}_{1}: \operatorname{ObD} \rightarrow \mathrm{ObC}, \mathcal{G}_{2}: \operatorname{Mor} \mathcal{D} \rightarrow \operatorname{Mor} \mathcal{C}$ and $\mathcal{G}_{1}(\mathcal{A}(n, m)):=\mathcal{A}(n, m)$. Let $d:=\left\{d_{h}: h>0\right\}$ be the family of difference operators corresponding to a parametric difference algebra from $\mathrm{ObD}$. Then we define $\mathcal{G}_{1}(d)$ by

$$
\left(\mathcal{G}_{1}(d)\right) \varphi:=\lim _{h \rightarrow 0} \frac{\left(d_{h} \varphi\right)-\varphi}{h} .
$$

$\mathcal{G}_{1}(d)$ is a well defined differential operator and it recovers $D_{\Sigma_{c}}$ - the differential operator of the continuous-time system that has been discretized. In fact, we have

$$
\begin{aligned}
\left(\mathcal{G}_{1} d\right) \varphi(x, U) & =\lim _{h \rightarrow 0} \frac{\varphi\left(x+h f(x, u), U+h c_{1}(U)\right)-\varphi(x, U)}{h} \\
& =\left.\frac{d}{d h}\right|_{h=0} \varphi\left(x+h f(x, u), U+h c_{1}(u)\right) \\
& =\frac{\partial \varphi}{\partial x}(x) \cdot f(x, u)+\frac{\partial \varphi}{\partial U}(x, U) \cdot \frac{\partial U+h c_{1}(U)}{\partial h} .
\end{aligned}
$$

Lemma 5.2. Let us assume that $\tau \in \operatorname{Mor} \mathcal{D}$. Then $\tau \in \operatorname{Mor} \mathcal{C}$, i.e is a homomorphism of the differential algebras.

Proof. From the assumption, $\tau \circ d_{h}=\tilde{d}_{h} \circ \tau$ for difference operators $d_{h}$ and $\tilde{d}_{h}$ and for any $h>0$. Then, continuity of $\tau$ implies that

$$
\begin{aligned}
\tau\left(\left(\mathcal{G}_{1} d\right) \varphi\right) & =\tau\left(\lim _{h \rightarrow 0} \frac{\left(d_{h} \varphi\right)-\varphi}{h}\right) \\
& =\lim _{h \rightarrow 0} \tau\left(\frac{\left(d_{h} \varphi\right)-\varphi}{h}\right) \\
& =\lim _{h \rightarrow 0} \frac{\tilde{d}_{h}(\tau \varphi)-(\tau \varphi)}{h}=\left(\mathcal{G}_{1} \tilde{d}\right)(\tau \varphi)
\end{aligned}
$$

This ends the proof.

Proposition 5.3. Let $\mathcal{F}$ and $\mathcal{G}$ be functors defined above. Then $\mathcal{F} \circ \mathcal{G}=\mathrm{id}$ and $\mathcal{G} \circ \mathcal{F}=$ id.

Proof. Because $\mathcal{F}_{1}(\mathcal{A}(n, m))=\mathcal{A}(n, m)$ and $\mathcal{G}_{1}(\mathcal{A}(n, m))=\mathcal{A}(n, m)$, it is enough to show that $\mathcal{F}_{1} \circ \mathcal{G}_{1}=$ id and $\mathcal{G}_{1} \circ \mathcal{F}_{1}=$ id on difference and differential operators, respectively. In fact, we have

$$
\begin{array}{r}
\left(\mathcal{F}_{1}\left(\mathcal{G}_{1} d\right)\right)_{h} \varphi=\varphi\left(\left(\mathrm{id}+h \mathcal{G}_{1} d\right) x,\left(\mathrm{id}+h \mathcal{G}_{1} d\right) U\right) \\
=\varphi\left(x+h \lim _{\tilde{h} \rightarrow 0} \frac{d_{\tilde{h}} x-x}{\tilde{h}} ; U+h \lim _{\tilde{h} \rightarrow 0} \frac{d_{\tilde{h}} U-U}{\tilde{h}}\right) \\
=\varphi\left(\left(\mathrm{id}+h D_{\Sigma_{c}}\right) x ;\left(\mathrm{id}+h D_{\Sigma_{c}}\right) U\right)=d_{h} \varphi
\end{array}
$$


for any $h>0$. On the other hand we have

$$
\begin{aligned}
\left(\mathcal{G}_{1}\left(\mathcal{F}_{1} D_{\Sigma_{c}}\right)\right) \varphi & =\lim _{h \rightarrow 0} \frac{\left(\mathcal{F}_{1} D_{\Sigma_{c}}\right) \varphi-\varphi}{h} \\
& =\lim _{h \rightarrow 0} \frac{\varphi\left(\left(\mathrm{id}+h D_{\Sigma_{c}}\right) x ;\left(\mathrm{id}+h D_{\Sigma_{c}}\right) U\right)-\varphi(x, U)}{h}
\end{aligned}
$$

which, by $(20)$, implies that $\mathcal{G}_{1}\left(\mathcal{F}_{1} D_{\Sigma_{c}}\right)=D_{\Sigma_{c}}$.

Let us assume conditions A1-A3. Now we can state the main result of this paper.

Theorem 5.4. Two continuous-time systems $\Sigma_{c}$ and $\tilde{\Sigma}_{c}$ are dynamically feedback equivalent if and only if their Euler's discretizations are h-dynamically feedback equivalent for every $h>0$.

Proof. Two continuous-time systems are dynamically feedback equivalent if and only if their differential algebras are isomorphic. Functor $\mathcal{F}$ transfers isomorphisms of the differential algebras (of the type $\mathcal{A}_{\Sigma_{c}}$ ) to isomorphisms of the corresponding parametric difference algebras (of the type $\mathcal{A}_{\Sigma_{d}}$ with the family of the difference operators $d_{h}$ ). This means that Euler's discretizations of the continuous-time systems are $h$-dynamically feedback equivalent for every discretization step $h$.

On the other hand, two discrete-time systems $\Sigma_{d}$ and $\tilde{\Sigma_{d}}$ that are Euler's discretizations with the step $h$ of two continuous-time systems are $h$-dynamically feedback equivalent if and only if their $h$-difference algebras are isomorphic. Functor $\mathcal{G}$ transfers isomorphisms of the parametric difference algebras (of the type $\mathcal{A}_{\Sigma_{d}}$ with the family of the difference operators $d_{h}$ ) to isomorphisms of the differential algebras (of the type $\mathcal{A}_{\Sigma_{c}}$ ). Hence, the continuous-time systems are dynamically feedback equivalent.

Example 5.5. Let $\Sigma_{c}$ be given by the equation

$$
\begin{aligned}
& \dot{x}=u \\
& \dot{y}=v \\
& \dot{z}=x v
\end{aligned}
$$

where $(x, y, z)$ is the state and $(u, v)$ is the control. We shall show that $\Sigma_{c}$ is dynamically feedback equivalent to the linear system $\tilde{\Sigma}_{c}$ :

$$
\begin{aligned}
& \dot{\tilde{x}}=\tilde{u} \\
& \dot{\tilde{y}}=\tilde{v} .
\end{aligned}
$$

Indeed, the dynamic transformations are given as follows:

$$
\tilde{x}=z, \quad \tilde{y}=y, \quad \tilde{u}=x v, \quad \tilde{v}=v,
$$

and

$$
x=\frac{\dot{\tilde{x}}}{\dot{\tilde{y}}}, \quad y=\tilde{y}, \quad z=\tilde{x}, \quad u=\frac{\dot{\tilde{u}} \tilde{v}-\tilde{u} \dot{\tilde{v}}}{\tilde{v}^{2}}, \quad v=\tilde{v} .
$$


Observe that the transformations are well defined if $\tilde{v} \neq 0$.

After Euler's discretization we obtain the following systems: $\Sigma_{d}^{h}$, given by equations

$$
\begin{aligned}
& x(k+1)=x(k)+h u(k) \\
& y(k+1)=y(k)+h v(k) \\
& z(k+1)=z(k)+h x(k) v(k)
\end{aligned}
$$

and $\tilde{\Sigma}_{d}^{h}$, given by equations

$$
\begin{aligned}
\tilde{x}(k+1) & =\tilde{x}(k)+h \tilde{u}(k) \\
\tilde{y}(k+1) & =\tilde{y}(k)+h \tilde{v}(k) .
\end{aligned}
$$

Let us consider transformations:

$$
\begin{aligned}
& \tilde{x}(k)=z(k), \quad \tilde{y}(k)=y(k), \\
& \tilde{u}(k)=x(k) v(k), \quad \tilde{v}(k)=v(k),
\end{aligned}
$$

and

$$
\begin{aligned}
& x(k)=\frac{\tilde{x}(k+1)-\tilde{x}(k)}{\tilde{y}(k+1)-\tilde{y}(k)}, \quad y(k)=\tilde{y}(k), \quad z(k)=\tilde{x}(k), \\
& u(k)=\frac{\tilde{u}(k+1) \tilde{v}(k)-\tilde{u}(k) \tilde{v}(k+1)}{h \tilde{v}(k) \tilde{v}(k+1)}, \quad v(k)=\tilde{v}(k) .
\end{aligned}
$$

Equations (25) define dynamic maps $\phi$ and $\psi$, and similarly equations (26) define dynamic maps $\tilde{\phi}$ and $\tilde{\psi}$. By Remark 3.1, choosing $H=c_{1}$ or $H=H_{h}$, we can extend $\psi$ and $\tilde{\psi}$ to $\Psi$ and $\tilde{\Psi}$, respectively. Then the maps $(\phi, \Psi)$ and $(\tilde{\phi}, \tilde{\Psi})$ give $H$-dynamic feedback equivalence of the discretized systems.

\section{Discretization and linearization}

Now we can apply the results of previous sections to the dynamic feedback linearization problem.

Corollary 6.1. A continuous-time system is dynamically feedback linearizable if and only if all its Euler's discretizations are h-dynamically feedback linearizable.

Proof. This follows from Theorem 5.4 and the fact that a linear continuous-time system is controllable if and only if its Euler discretization is also controllable.

Following Jakubczyk (1992) and Pawłuszewicz (1998) we say that a differential algebra $(\mathcal{A}, D)$ (a difference algebra $(\mathcal{A}, d)$, respectively) is free if there exist $\omega_{1}, \ldots, \omega_{r} \in \mathcal{A}$ such that

1. for any function $\nu: \mathbb{R}^{k} \rightarrow \mathbb{R}$ of class $C^{s}$ there holds $\nu \circ W \equiv 0 \Rightarrow \nu \equiv 0$, where 
$W=\left\{D^{j} w_{i}\right\}_{\substack{i=1, \ldots, r \\ j=0,1, \ldots}}$, (respectively $W=\left\{d^{j} w_{i}\right\}_{\substack{i=1, \ldots, r \\ j=0,1, \ldots}}$ )

2. for any $\varphi \in \mathcal{A}$ there exists a function $\nu: \mathbb{R}^{k} \rightarrow \mathbb{R}$ of class $C^{s}$ such that $\varphi=\nu \circ W$.

Functions $\omega_{1}, \ldots, \omega_{r}$ are called free generators of the differential algebra $(\mathcal{A}, D)$ (respectively of the difference algebra $(\mathcal{A}, d)$ ).

Jakubczyk (1992) proved that the continuous-time control system $\Sigma_{c}$ is dynamically linearizable if and only if its differential algebra $\mathcal{A}_{\Sigma_{c}}$ is free. Pawłuszewicz (1998) showed a similar result for discrete-time case.

Corollary 6.2. Let us consider system $\Sigma_{c}$ and its Euler's discretiztions. Then $\left(\mathcal{A}(n, m), D_{\Sigma_{c}}\right)$ is a free differential algebra if and only if $\left(\mathcal{A}(n, m), d_{h}\right)$ is free for every $h>0$.

Acknowledgement. This work was supported by KBN under the Białystok Technical University grant No. W/IMF/1/04.

\section{Bibliography}

Arapostatis, A., JAkubczyk, B., et al., 1989, The effect of sampling on linear equivalence and feedback linearization. Systems \& Control Letters, 13, 373-381.

Bartosiewicz, Z., Jakubczyk, B. and Pawłuszewicz, E., 1994, Dynamic feedback equivalence of nonlinear discrete-time systems. Proc. First Internat. Symp. on Mathematical Models in Automation and Robotics, Miedzyzdroje, Poland, 1-3 September, Tech. Univ. of Szczecin Press, 37-40.

Celikovský, S., 1995, On the relation between nonsmooth linearization of continuous and discrete time systems. Proceedings of 3rd European Control Conference, Rome, Italy, September, 643-648.

FLIESS, M., 1986, Some remarks on nonlinear invertibility and dynamic state feedback. In C.I. Byrnes and A. Lindquist (eds) Theory and Applications of Nonlinear Control Systems (Amsterdam: Elsevier), 115-123.

FLIESs, M., 1987, Esquisses pour une theorie des systems non lineaires en temps discret. Rediconti del Seminario Matematico, Universitàe Politecnico Torino, Fasciolo speciale, 55-67.

FliEss, M., 1990, Automatique en temps discret at algèbre aux différences. Forum Mat., 2, 213-232.

Fliess, M., Lévine, J., Martin, P., and Rouchon, P., 1992, Sur les systemes non liné aires differentiellment plat. Comptes Rendus Acad. Sci. Paris, I-315, 619-624.

Grizzle, J.W., and Kokotovic, P.V., 1988, Feedback linearization of sampleddata systems, IEEE Trans. on Autom. Contr., 33, 857-859.

JAKUBCZYK, B., 1992, Remarks on equivalence and linearization of nonlinear systems. Proceedings of the 2nd IFAC NOLCOS Symposium, Bordeaux, France, 393-397.

JAKUBCZYK, B., 1993, Invariants of dynamic feedback and free systems. Proceedings of the second European Control Conference ECC'93, Groningen, The Netherlands,1510-1513. 
KaZAntZis, N., and Kravaris, C., 1997, System-theoretic properties of sampleddata representations of nonlinear systems obtained via Taylor-Lie series. Int. J. Control, 67, 997-1020.

Kотта, Yu. R, 1993, Synthesizing nonlinear systems with specified inputoutput mappings. Sampling effects. J. Comput. Systems Sci. Internat, 31, 66-76

Monaco, S., and Normand-Cyrot, D., 1988, Zero-dynamics of sampled nonlinear systems. Systems 83 Control Letters, 11, 229-234.

PawŁuszewicz, E., 1998, External linearization of discrete-time systems. Proceedings of IV Int.Conf. on Difference Equations and Applications ICDEA'98, Poznań, Poland, 27-31 August, 295-298.

PawŁuszewicz, E., and Bartosiewicz, Z., 2000, Euler's discretization and dynamic equivalence of nonlinear control systems. In A. Isidori, F. LamnabhiLagarrigue, and W. Respondek (eds) Nonlinear Control in the Year 2000, Lecture Notes in Control and Information Sciences 259, vol.II, (New York: SpringerVerlag), 183-191.

Pomet, J.-B., 1995, A differential geometric setting for dynamic equivalence and dynamic linearization. In Geometry in Nonlinear Control and Differential Inclusions, Banach Center Publication, vol.32, Warsaw, 319-339. 